This item was submitted to Loughborough's Research Repository by the author.

Items in Figshare are protected by copyright, with all rights reserved, unless otherwise indicated.

\title{
Case study of knowledge management implementation in a medium-sized construction sector firm
}

PLEASE CITE THE PUBLISHED VERSION

http://dx.doi.org/10.1061/(ASCE)ME.1943-5479.0000109

PUBLISHER

(C) American Society of Civil Engineers

VERSION

AM (Accepted Manuscript)

LICENCE

CC BY-NC-ND 4.0

\section{REPOSITORY RECORD}

Tan, Hai Chen, Patricia M. Carrillo, and Chimay J. Anumba. 2019. "Case Study of Knowledge Management Implementation in a Medium-sized Construction Sector Firm". figshare. https://hdl.handle.net/2134/11315. 
This item was submitted to Loughborough's Institutional Repository (https://dspace.lboro.ac.uk/) by the author and is made available under the following Creative Commons Licence conditions.

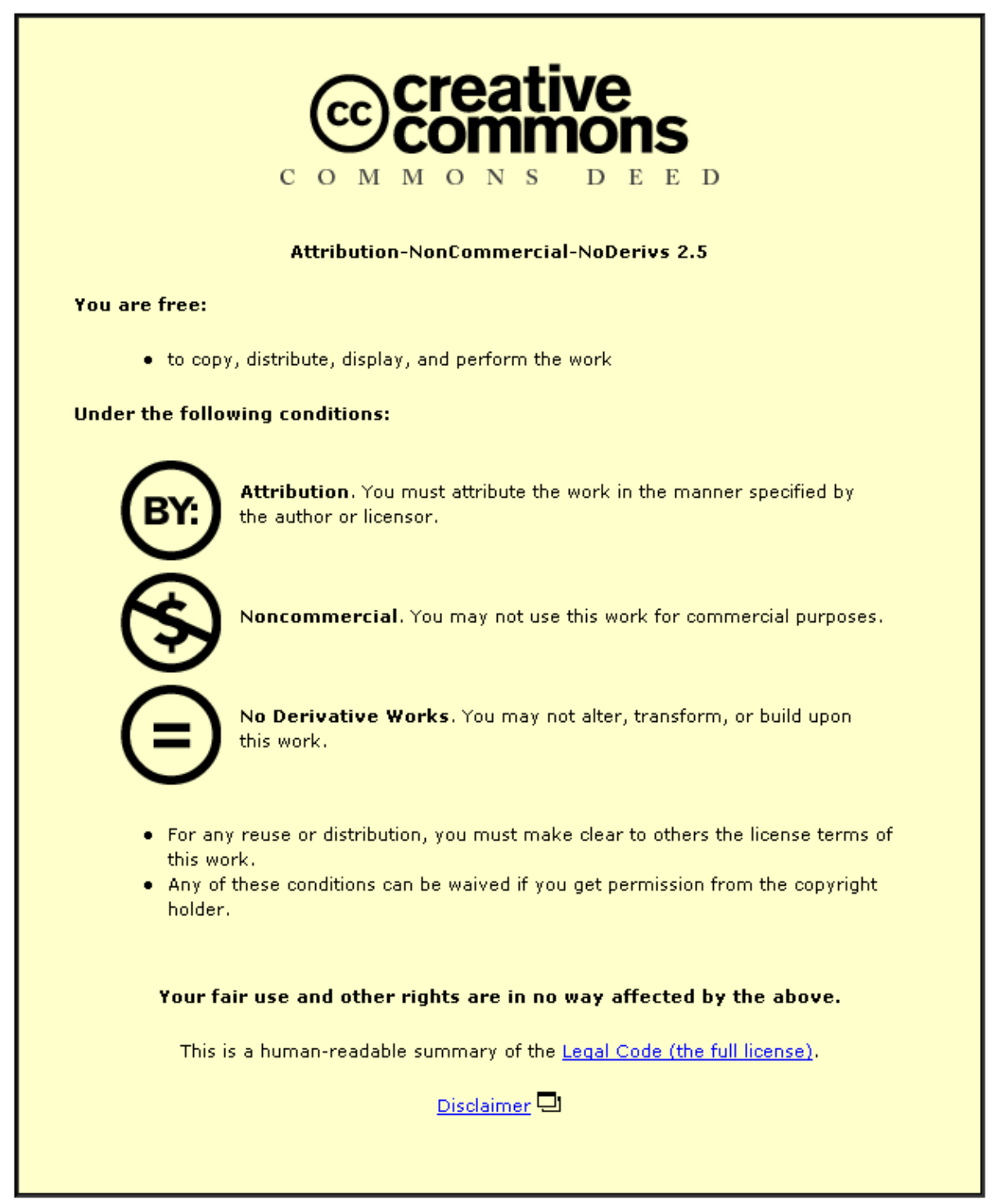

For the full text of this licence, please go to: http://creativecommons.org/licenses/by-nc-nd/2.5/ 


\section{Case Study of Knowledge Management Implementation in a Medium-Sized}

\section{Construction Sector Firm}

Hai Chen $\operatorname{Tan}^{1}$, Patricia M. Carrillo ${ }^{2}$ and Chimay J. Anumba ${ }^{3}$

${ }^{1}$ Assistant Professor, Dept. of Built Environment, Universiti Tunku Abdul Rahman, 53300 Kuala Lumpur, Malaysia; PH (60) 03- 41079802; FAX (60) 03-41079803; Email: tanhc@utar.edu.my

${ }^{2}$ Professor, Dept. of Civil and Building Engineering, Loughborough Univ., LE11 3TU Leicestershire, UK; PH (44) 1509 222634; Email: p.m.carrillo@lboro.ac.uk

${ }^{3}$ Professor and Head, Dept. of Architectural Engineering, The Pennsylvania State Univ., PA 16802, USA; PH (1) 814-865-6394; FAX (1) 814-863-4789; Email: anumba@engr.psu.edu

\section{Abstract:}

The transient nature of project teams and the competitive environment in which construction organizations operate require an effective mechanism to manage and reuse the knowledge created in the projects without straining its resources. A methodology for 'live' capture and reuse of project knowledge was developed for the purpose and encapsulated into a software tool called Capri.net. A study was conducted of a medium-sized UK-based construction sector firm to investigate the use of Capri.net as the central strand of its KM framework. Capri.net was subsequently designed to integrate with a staff profile system and an e-forum. The new Capri.net-based system was intended to bridge the communication gap between the company's geographically dispersed offices by connecting the people together and hence facilitating the timely sharing of both tacit and explicit knowledge. Top management's commitment was found vital in the successful implementation of 
knowledge management and the nurturing of the desired supportive knowledge sharing culture. To get the most through the implementation of knowledge management, the various knowledge management practices and relevant information systems of a construction organization need to be strategically integrated to improve the location, capture and sharing of critical knowledge.

\section{CE Database subject headings:}

Knowledge-based systems; Construction management, Information systems; Project management; Information technology (IT). 


\section{Introduction}

Knowledge management has been receiving much attention from the construction sector in recent years judging from the number of research conducted, numerous relevant articles and books published, and also the availability of reported success cases at varying degrees on its implementation in construction organizations. The importance of knowledge management (KM) has been recognized and is often related to the emergence of knowledge economy. In the knowledge economy, knowledge is said to sideline both capital and labor to become the 'sole factor of production' (Drucker 1993) which influences an organization's ability to sustain its competitive advantage (Nguyen et al. 2009). In order to remain competitive, the critical innovation and ability to respond to customers quickly are also dependent on the amount of knowledge available. This, in turn, relies on $\mathrm{KM}$ to provide both the platforms and processes to facilitate the desired collaboration in the innovation process, and to ensure the availability as well as accessibility of both tacit and explicit knowledge required (Plessis 2007). Furthermore, organizations also count on KM to retain a copy of organizational memory of its collective knowledge to address the potential knowledge loss problem due to the high mobility of workforce, or the so-called "corporate amnesia" (Dalkir 2007).

The management of an organization's knowledge is challenging owing to the different nature of knowledge, the various barriers to the successful implementation of KM (Chinowsky and Carrillo 2007), and the need to strategically customize one to suit the context of individual organization. Knowledge is predominantly distinguished as either explicit or tacit (Nonaka and Takeuchi 1995) with the latter notoriously difficult to articulate and manage, whereas KM is the purposeful and systematic management of knowledge and the associated processes and tools with the aim of realizing fully the potential of knowledge in making effective decisions, solving problems, facilitating innovations and creativity and achieving competitive advantage at all levels (Kebede 2010). The current approach to KM is reflected by the two main perspectives through which KM is defined, namely the information systems and human resource management perspectives. From the former, 
$\mathrm{KM}$ is concerned with the use of information and communication technology (ICT) to facilitate the capture, deployment, access and reuse of information and knowledge (O'Leary, 2001), whereas the human resource management perspective emphasizes the establishment of means to motivate and facilitate knowledge workers to develop, enhance and use their knowledge in order to achieve organizational goals (Beijerse 1999). However, leveraging knowledge through ICT alone is often hard to achieve (Siemieniuch and Sinclair 1999; Walsham 2001; Rollett 2003) as there are human, cultural and organizational issues such as reluctance to share knowledge which are not readily resolved by ICT. Conversely, a purely human resource management approach is not going to benefit from the faster, cheaper and broader source of data and means of communication to enable people to generate and share knowledge offered by ICT. Therefore, an integrated approach of KM combining information systems (technology) and human resource management (people) synergized by the benefits of both perspectives is likely to be a more viable option (Davenport 1998).

The paper begins with discussions on knowledge management in construction and the shortcomings of current practice. It then describes the importance of the methodology for 'live' capture and reuse of project knowledge and the software prototype (Capri.net), which was developed to implement the methodology. Subsequently, the details of the existing knowledge management practice and plan formulated for integrating the Capri.net methodology with other systems in the case study company (hereinafter referred to as Company A) are presented. The paper concludes with a discussion on the potential impacts and expected benefits of the integrated system.

\section{Knowledge Management in Construction}

Construction is a project-based industry. Therefore, it is not surprising that most of the knowledge of the construction industry is generated in projects during the process to deliver a custom-built facility in accordance with the client's requirements and business objectives. The ability to manage the 
knowledge generated from the projects not only can help to prevent the 'reinvention of the wheel' and the repetition of similar mistakes, but also serves as the basis for innovation, overall improvement and sustaining competitive advantage. However, the ability to learn from within and across projects are critical but difficult to achieve. This is often due to the tight timeframe of construction projects, and the lack of sufficient resources and standard work processes for managing project knowledge.

There are a variety of $\mathrm{KM}$ tools commonly used for the capture and sharing of knowledge in construction, which can be broadly categorized as KM technologies (IT-tools) and KM techniques(non-IT tools) (Al-Ghassani 2003). These include Groupware, expert or skills directory, custom-designed software, forum, post project reviews (PPRs), communities of practice (CoPs), etc. Among the KM tools, PPRs and CoPs are the most widely used approach for capturing and sharing of knowledge in construction (Orange et al. 1999; Carrillo et al. 2002). The main shortcoming of PPRs owing to the timing at which it is conducted has lend itself to knowledge loss problem as many important lessons and learning might have been forgotten (Kamara et al. 2003), project participants are already preoccupied with new project and hence reluctant to devote their time for the purpose (Kartam 1996), emphasis on negative feedback (Kotnour and Vergopia 2007) and the insufficient willingness to learn from mistakes (Schindler and Eppler 2003). The significance of CoPs towards KM is generalized by Saint-Onge and Wallace (2003) as providing a platform for their members to pool their expertise, experience and ideas, and to find solutions. However, the efficacy of knowledge sharing and transfer in CoPs is deeply influenced by the norms of reciprocity - 'you help me and I will help you' - and the level of trust generated amongst the community (Newell et al, 2002). Furthermore, CoPs do not proactively capture knowledge. Knowledge is mostly shared in CoPs in response to the request for assistance from members or through discussion, instead of proactively pushed to relevant parties once it is discovered elsewhere in the organization. Simply put, if a question is never asked, the knowledge pertaining to the question is less likely to be shared. Consequently, staff might not be aware of the existence of certain knowledge within an organization 
which hinders the reuse and the ability to leverage from the knowledge. "If TI only knew what $\mathrm{TI}$ knows" stated by Jerry Junkins (O'Dell and Grayson 1998), the late CEO of Texas Instruments, is the typical frustration of an organization unable to tap into its unknown and hence not-being-shared knowledge. In addition, there are also other main challenges faced in maintaining CoPs, such as how to retain members and facilitate their knowledge-sharing continuance intention (Fang and Chiu 2010).

Moreover, the fact that the project team either splits up or moves to another project after the completion of a project is also not conducive to the capture, sharing and reuse of knowledge across projects. As a result, knowledge loss is not an uncommon issue. The high staff turnover, which was 13.5\% in 2010 in the UK (CIPD 2010), has also further aggravated the knowledge loss problem.

\section{The Importance of 'Live' Capture and Reuse of Project Knowledge}

In order to overcome the limitations in current industry practice on the capture and reuse of knowledge, it is necessary that learning from projects is captured and shared 'live' while it is being executed, and presented in a format that will facilitate its reuse both during and after the project (Kamara et al. 2003). The 'live' capture and reuse of project knowledge methodology facilitates the capture of project knowledge as soon as the knowledge is created or identified (i.e. 'live') and subsequent sharing of the knowledge instantly with the aid of information and communication technology to avoid persistent knowledge loss problem due to time lapse and other constraints. The imperative of 'live' capture of knowledge is supported by the survey of organizations involved in PFI (Private Finance Initiative) projects where the 'live' capture of knowledge is noted as crucial by $76 \%$ of construction organizations and $70 \%$ of client organizations (Robinson et al. 2004). Furthermore, the need for 'live' capture of knowledge is also being indirectly addressed by Whetherill et al. (2002). They assert that a construction organization's only sustainable advantage lies in its capability to 
learn faster than its competitors and the rate of change imposed by the external environment, and that there is a need to 'integrate learning within day-to-day work processes'. The strategy of the 'live' capture proposed by Kamara et al. (2003) attempts to address the cross-organizational knowledge transfer issues through particularly collaborative learning and learning histories and to facilitate 'live' capture and reuse of knowledge through Web-based technology respectively. The potential benefits of 'live' capture and reuse of project knowledge are as follows:

- $\quad$ Prevention of knowledge loss due to time lapse in capturing the knowledge. Knowledge created needs to be captured as soon as possible as Ebbinghaus's (1885) study reveals that the percentage of human memory retained on a set of data depletes over time. Furthermore, the probability of forgetting an event (which may include the knowledge learned) is increasing as time elapses (Linton 1975);

- Maximization of the value of reusing the knowledge captured through 'live' reuse. The true benefit of capturing knowledge comes only when the knowledge is being reused (McGee 2004), particularly if the knowledge is being reused 'live' after it has been captured. This is obvious when the benefit accrued through reusing the knowledge is time-related (e.g. leading to a saving of $\$ x$ per day);

- $\quad$ Help to seize every knowledge reuse opportunity. Knowledge captured may have limited number of events for reuse and hence has to be disseminated for reuse as soon as possible (i.e. 'live') before such events diminish. Using Three Mile Island (TMI) incident as an example, Mc Gee (2004) points out that the lesson learned about a faulty relief valve in a nuclear reactor was not shared with the operator of TMI although the same type of valve was used. The lesson learned was hence not reused. No action was taken to replace the faulty valve which subsequently led to the TMI incident when the valve failed; 
- $\quad$ Provide knowledge that can be utilized at the operation and maintenance stages of the assets' lifecycle;

- $\quad$ Facilitate the reuse of collective learning on a project by individual firms and teams involved in its delivery. More insights are likely to be captured in the collaborative environment, as each of the members in the project team knows only bits of the whole story about the project (Kerth 2000); and

- Benefit the client organizations with enriched knowledge about the development and construction of their assets. This will contribute to the effective management of facilities and the commissioning of other projects. In the longer term, clients will benefit from the increased certainty with which construction firms can predict project outcomes.

\section{The 'Live' Capture and Reuse of Project Knowledge Methodology}

A methodology for the 'live' capture and reuse of project knowledge was developed based on the findings from the case studies involving semi-structured interviews with 18 senior staff from six companies. The companies were the partners of the CAPRIKON research project that aimed to develop the methodology. The positions of the interviewees ranged from Group Knowledge Manager to Company Partner to ensure that a comprehensive view was obtained. Background information on the case study companies is presented in Table 1. The interviews focused on the investigation of the current practice for knowledge capture and the associated shortcomings, and the identification of the requirements for the design of a system for knowledge capture and reuse by various end users of project knowledge. The shortcomings of current practice and end-users' requirements identified were then analyzed to formulate the methodology. The methodology is subsequently encapsulated into a Web-based application called Capri.net given that the main strengths of Web-based technology are the capability to connect distant offices together, provide 
fast access and location of knowledge captured, facilitate sharing of knowledge and provide huge knowledge storage space. Web-based technology plays a critical role in facilitating the 'live' capture and reuse of project knowledge for users at different locations in real time. Furthermore, it also enables a standard format for representing knowledge to be specified, a mechanism for validating the accuracy of knowledge to be created, and saving on staff time as the additional workload created for the purposes can be handled by the system.

[Insert Table 1 here]

Another important concept for the development of the methodology is the existence of three dimensions of reusable project knowledge. It was discovered that reusable project knowledge often exists as a mixture of tacit and explicit knowledge, rather than as distinctive tacit or explicit knowledge alone. As explicit knowledge is comparatively easier to be transferred and shared, the methodology is designed to explicate tacit knowledge into explicit knowledge as far as possible. For the remaining tacit knowledge which is really difficult to be explicated, means will be provided to connect the people with the knowledge to those who need the knowledge.

The methodology, as depicted in Fig. 1, comprises:

- $\quad A$ Web-based knowledge base: This is where the Project Knowledge File (PKF) of a project is stored. A PKF contains relevant project information and project knowledge that can be reused both during the execution (e.g. in subsequent phases) and after the completion of the project. A PKF is similar to the Health and Safety File (HSF) under the Construction (Design and Management) (CDM) Regulations in the United Kingdom. The difference being that HSF is a project record which focuses on health and safety (HSE 2007), whereas the PKF targets reusable project knowledge.

[Insert Figure 1 here] 
- A Project Knowledge Manager (PKM): This is a role, normally charged to a project manager or other designated person, to manage the knowledge base (i.e. the development of a PKF for a project) and the Integrated Workflow System.

- $\quad$ An Integrated Workflow System: This delineates, executes and monitors the mechanism for the capture, validation and dissemination of the project knowledge captured. A PKM may configure the Integrated Workflow System to suit individual requirements of the project.

The methodology is designed to capture important reusable project knowledge from: (1) project meetings/reviews, (2) individual submissions, and (3) rationale for making changes to documents. This is because it was found that the generation of new knowledge in learning organizations might either involve the interactions among a group of people (e.g. in project meetings) or an individual only (e.g. problem solving which may involve only an individual). The mechanisms for capturing knowledge from the three sources are as follows:

\section{- (a) Capture of knowledge from project meetings/reviews}

The PKM will be responsible for including the capture of reusable project knowledge as an agenda item in the routine project meetings/reviews. During the meetings/reviews, the learning captured since the previous meeting/review is discussed and the details agreed. If the system is accessible during the meeting/review, the designated person (who is normally the PKM) may enter the approved knowledge directly into the knowledge base in the specified format. Otherwise, the designated person may transfer the record into the system at a later time.

\section{- $\quad$ (b) Capture of knowledge from individuals}

All knowledge workers involved in the project will be assigned a login name and password to access the system. This allows them to enter their knowledge into the system once knowledge is created or identified (i.e. 'live'), or at anytime which is convenient to them. The system will send routine email reminders to the users who have opted to receive them. 
- (c) Capture of the rationale for making changes to documents

The findings of the case studies revealed that the rationale for making changes to project documents (such as engineering drawings) is important reusable project knowledge. If there is a project document for which the number of changes made to it is well above average, a procedure for the capture of the rationale for making the changes to the document can be invoked by the PKM. The author of the project document will be requested to provide the necessary details to the system. Similar to the knowledge submitted by individuals, the rationale for changes made to documents will be subject to validation before it can be disseminated.

It is important that the knowledge captured in the course of a project is as accurate and reliable as possible. This requires that it is validated. The knowledge captured from a group (in meetings and reviews) is deemed to have been validated whereas the knowledge submitted by individuals may need to be validated prior to reuse. However, at the organization's discretion, the validation process may be omitted for the knowledge submitted by their experts and very experienced staff. The validation mechanism is triggered once new knowledge is entered into the system by individuals. There are four modes available for validation process, i.e. no validation required, validation based on the average ratings given, validation based on the majority's view, and validation based on the comments given.

The 'live' sharing of project knowledge is achieved by proactively and automatically emailing users to notify them about the addition of a new knowledge item. The users should be notified of the changes made to the status of a knowledge item in the system. In addition, knowledge is also made available for access online once it has been added into the system.

An evaluation that involved 10 participants from the four case study companies (i.e. Companies A, B, $\mathrm{D}$ and $\mathrm{E})$ was conducted to evaluate the performance of the methodology. The questionnaire designed for the purpose evaluated the methodology on four aspects, namely capture of knowledge, 
representation of knowledge, sharing/reuse of knowledge, and ease of use. The participants were requested to provide their answers to the questions using a rating scale from 1 (very poor) to 5 (excellent). The methodology scored an average 3.9 out of 5.0 in the evaluation. The participants were very satisfied with the ability of the system to facilitate the 'live' capture of project knowledge and its ease of use, which scored an average rating of 4.3 (see Tan et al, 2010).

\section{Research Methodology}

Company A was one of the collaborating organizations actively involved in the development of the Capri.net methodology. As Company A intended to develop an improved system for capturing reusable project knowledge from its projects, it wished to explore how the methodology can be adopted with necessary modification in order to integrate with its other KM systems and activities. For the purpose, one of the researchers had spent a year attached to Company A. The researcher observed and attended Company A's board of directors' meetings, routine project meetings and communities of practice's meetings, and studied the features and functions of Company A's various IT systems and practices that might assist in KM activities. In addition, the researcher conducted nine semi-structured interviews involving 19 of the Company A's staff from the position of Managing Director to general administration staff in order to gain more insights into the issues that have a bearing on KM. The interviewees selected were either senior staff or have served the company for many years, for their familiarity with the operation of the company and ability to provide valuable input into the development of the KM system. Five of the interviews were conducted in a focus group, as requested by the interviewees, and others were individual interviews. The interviews focused on the followings:

- $\quad$ Suggestions for improving the existing KM system and practice; and 
- The issues of concern for the implementation of $\mathrm{KM}$ and the integration of Capri.net methodology with existing KM system.

A roadmap detailing the strategy to implement $\mathrm{KM}$ and the way to integrate the methodology with Company A's other existing systems was subsequently prepared. The potential impacts and benefits to be brought about by this were also discussed.

\section{The Case Study Company}

\section{Company Background}

The case study company (i.e. Company A) is a holding company with a group of smaller construction companies in the UK. Within Company A there is a construction arm, a property development company and a design consultancy with a total of approximately 850 staff employed across its various offices in the UK. Company A is privately owned; the Chairman cum CEO and his brother are the largest shareholders with a number of other minority shareholders. Company A started its KM implementation in 2000 but had no dedicated staff, other than one of its managing directors who acted as the champion for KM.

\section{Implementation of $K M$}

Securing the commitment of the top management has been recognized as both the key and the main challenge for the successful implementation of knowledge management in an organization (Trussler 1999). It is critical as the implementation of KM often entails the commitment of resources (e.g. staff time and budget allocation) over a period of time from the top management, whose commitment frequently requires good justification substantiated by a cost and benefit analysis. Whilst the cost for implementing $\mathrm{KM}$ can easily be estimated, the resultant benefits are 
unfortunately not always tangible and measurable. For Company A, however, this notorious challenge is not an issue as the plan to implement $\mathrm{KM}$ was a top down decision initiated by the Chairman/CEO. Hence, the commitment is automatically assured.

Although lacking in internal KM expertise, Company A was firm with its decision and resorted to a logical first move by engaging the service of an external consultancy to study its existing internal IT resources and the KM related business processes prior to launching any plan. The consultant recommended for: (1) full audit of the present IT systems on their functions and shortcomings that need to be addressed; (2) an audit of the procedures for conducting post project reviews; (3) a study to investigate Company A's senior staff views as to how KM can help in the execution of their tasks to be conducted; and (4) an associated action plan.

Subsequently, one of the managing directors of Company A's businesses was assigned the responsibility to champion the KM implementation plan. Resources were allocated for the participation in nationwide forums and research projects related to KM with the aim to bring back useful ideas and insights for the purpose, other internal KM activities and making improvement to the IT systems as recommended by the consultant. At the stage, the key KM activities and systems in Company $\mathrm{A}$ included its communities of practice, intranet system, post project reviews, and the creation of an electronic and physical meeting space for staff to interact with each other with the aim to trigger knowledge sharing and creation. These are described in following sections.

\section{Communities of Practice}

A community of practice is a living context that can give newcomers access to expertise and also to invite a personal experience of engagement by which to incorporate that competence into an identity of participation (Wenger 2004). Company A's KM practice is characterized by its eight communities of practice (CoPs) for estimating, key account management, surveying, key 
performance indicators, design management, administration and secretarial, contract management, and planning and programming respectively, but only the first three are active. The memberships of the CoPs were predetermined as not everyone from a practice would be automatically admitted as a member. Instead, it was confined to the very senior staff from the practice, with some nominated members from other stakeholding functions. For instance, the Director of Information Systems and a senior key account manager were among the nine members of Estimating CoP. Meetings of a CoP were organized by its champion who would also announce to other members the program or agenda of the next meeting for them to decide their attendance. There were also formal sessions organized purposely for sharing knowledge on special topics determined by the CoPs which were open to other non-members.

Company A was found suffering from the vertical silos of knowledge problem as, for instance, the estimators are not all co-located and belong to different departments. Knowledge sharing only happened vertically between the estimators across the ranks within the same department but not across departments. The existence of CoPs might have helped to address the vertical silos of knowledge problem for a practice, but only at the upper level for the senior-positioned estimators who participated in the CoP. The lower-positioned estimators still failed to benefit from the practice. Furthermore, the absence of a member who is an expert of an issue discussed in the CoP's meeting would also have impacts on the value of knowledge created. Similarly, a member's inability to attend a meeting due to other more pressing commitments also implies that certain important knowledge created is less likely to come to his attention.

\section{Capturing Knowledge from Routine Project Meetings}

As previously mentioned, PPRs are known for their knowledge loss problem due to the time lapse in capturing knowledge after the completion of a project. Company A attempted to address this issue 
through capturing knowledge from the routine weekly/bi-weekly project meeting for the earmarked projects. These were normally chosen either due to the scale or complexity. An agenda item called "knowledge management" is made mandatory where the 10-20 minutes slot allocated allows participants to bring up any positive or negative learning that is worth the attention. The learning was recorded as part of the minutes of the meeting in a very concise way.

There was no established mechanism to locate the learning captured in the minutes of the meetings for reuse. The minutes was submitted to the office, subsequently saved in the company's intranet and stopped there. It required one to read through the minutes one by one in order to identify some relevant reusable learning from a project, which did not help to encourage its usage. The practice to shrink the key learning captured into point form is also too brief for understanding and the context within which the learning can be reused is also lost.

\section{Intranet}

Company A's intranet is based on the Lotus ${ }^{\mathrm{TM}}$ system. The system allows a number of "space", which are essentially Web pages with database support, created for some of the CoPs within the intranet. Minutes of meetings, records and standard procedures were archived in the space. The documents archived, albeit useful and important for daily operations, are not all of the knowledge rich types. There were occasional articles prepared purposely for sharing knowledge on certain topics being uploaded into the space. However, access to the space owned by different CoPs and also the right to upload documents were restricted to their respective members only. Other than these, Company $\mathrm{A}$ also possessed its own email server, and a Lotus ${ }^{\mathrm{TM}}$-based system for organizing meeting as well as an extranet.

\section{Knowledge Café}


Company A used to have a few offices housing different departments at various locations in the city where its head office is located. This had inhibited the desired interactions among staff for knowledge sharing. When this study was conducted, Company A was starting to move all its administration offices into the new head office in the same city. Thus face-to-face interactions among staff from previously segregated offices should not have been a problem. However, management contended that as the staff were still grouped together in the new office according to job functions their social circle within the company would likely be confined to those sitting in vicinity.

Consequently, a Knowledge Café was created in the new Company A office with a space for all the staff to have lunch, hold informal meetings and socialize. The rationale for creating the Knowledge Café is coincidentally very similar to that of "ba" advocated by Nonaka and Konno (1998). Drinks making machines and access to water were made only available at the Knowledge Café located at the ground floor, deliberately planned in such a away to increase the chances of the staff at the multi-storey building to interact with each other and hence also the possibility of knowledge sharing and creation.

\section{Managing Knowledge of Who Knows What}

Knowledge of who knows what enables one to identify the existence of a particular type of knowledge and who possesses it within the organization for knowledge sharing purpose. Company A's main approach for managing this knowledge leveraged on the fact that most of its staff stay long with the company which allows them, throughout the time, to establish their own network of people and learn about what each other know. Company A contended that this knowledge resides in the head of its long serving senior staff and was critical for facilitating knowledge sharing. There was a CV section in Company A's central datastore but it does not cover all its staff. Furthermore, it also 
lacked the contact details and the expertise which are essential to facilitate interactions among staff for knowledge sharing. Hence, the only way to access this knowledge was to pose the query to the long serving staff who, ideally, can refer one to the right person.

Company A's approach for managing this critical knowledge lends itself to a number of problems: (1) this knowledge leaves together with the person when they retire or move to another company; $(2)$ it is susceptible to loss as human memory is not perfect; (3) access to the knowledge is subject to the availability of the person who possesses it; (4) the process to accumulate this knowledge is time consuming; and (5) one's possession of the knowledge is restricted by their exposure, people who they know, as well as how observant they are. Given that Company A has around 850 staff, it is almost impossible for anyone to know everything about "who knows what" within the company. Whilst this approach may have worked well so far in Company A, there is still room for improvements in view of the aforementioned issues that need to be addressed.

\section{Factors Affecting Company A's KM Practices}

For the development of a new KM framework for an organization, it is prudent to build upon the strengths of its existing practices with the identified weaknesses also being addressed. A number of issues, which were found either to have influenced the success of the implementation of KM in Company A or required close attention when designing the new KM framework, were as follows:

- Top management's commitment: Based on the top management's track records on other policies, such as heading towards 50:50 female/male staff ratio in 2011 which had already shown some convincing result, Company A's staff had only little doubt, if at all, of its commitment towards the implementation of KM. Although a number of researchers have argued for the importance of bottom-up initiative for the implementation of $\mathrm{KM}$ in an organization, the top-down approach worked in Company A and helped shorten the time required for the purpose. Instead of spending 
time and efforts to obtain the commitment from the top management to implement $\mathrm{KM}$, which is typical, the time saved had been utilized for planning and executing actual KM activities.

- Unique culture: The unique culture of Company A where most of its staff stay very long (with some are in fact the second generation serving the same company), the existence of a friendly atmosphere and the commitment demonstrated by the top management had contributed to the reduction of potential rejection for $\mathrm{KM}$. These were also found positive to the nurture of a supportive knowledge sharing culture in Company A.

- Rewards: Whilst supportive of the company's KM plan, generally Company A's staff were concerned with the potential increased additional workload and time spent on KM activities. Albeit admitting that KM in some instances had helped them in discharging their official duties, they pointed out that unfortunately their involvement in KM activities was not counted for in the annual performance appraisal.

- Additional resource requirements and constraints: As Company A was at the time in the midst of integrating its legacy finance system with a new system, the development of IT system for KM purpose might have to be carried out in phases. Furthermore, Company A's existing IT infrastructure and $\mathrm{KM}$ systems were based on Lotus ${ }^{\mathrm{TM}}$ products, whilst the Capri.net prototype was developed using ASP.net technology. If the prototype was to be further developed and integrated

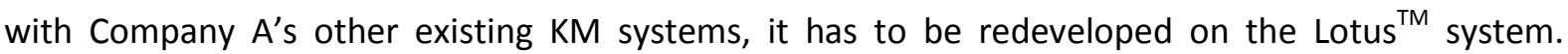
Fortunately with the existing IT infrastructure and a team of in-house IT specialist available, these were not regarded as a barrier by Company A.

- IT support: Company A's KM implementation strategy was primarily people-based with some IT support, which sometimes was found insufficient. Whilst sharing of knowledge happened in the CoPs and routine project meetings, the access is restricted to only the participants and the lack of a more systematic mechanism or IT system to share the knowledge captured to the wider audience. In 
addition, the location of reusable project knowledge captured in the minutes of routine project meetings had still to be done by manually skimming through the files. The online databases and "space" created for CoPs were not interconnected, which led to the stovepiping problem and reduced efficiency.

In view of the above issues, there is a need for more holistic approach to redesigning Company A's KM system to allow its invaluable knowledge, either residing in the heads of its staff or captured in documents/electronically, to be accessed with ease and hence fully harnessed. The outcomes of the nine semi-structured interviews conducted were then combined with the findings from the study conducted on Company A's KM practice and system for the development of the integrated system. A proposed new framework with roadmap and strategy for implementation were prepared and was subsequently accepted by Company A. The new framework comprised mainly the following:

- $\quad$ Staff Profile;

- $\quad$ Custom-designed e-forum for CoPs; and

- $\quad$ Capri.net system, which will be integrated with both the above systems.

\section{Adaptation of Capri.net Methodology for Company A}

The Capri.net system, which had to be re-developed on Company $\mathrm{A}^{\prime} \mathrm{s}$ Lotus ${ }^{\mathrm{TM}}$ system, served as the backbone of the overall framework. Staff profile would need to be developed as an integral component within the Capri.net system, whilst the e-forum is designed to be an external system with linkage to and from Capri.net system. This is depicted in Fig. 2. For staff profile, the Capri.net system already has a feature that captures some of the user's personal and contact details to ease the communication. Therefore, the staff profile for managing the knowledge of who knows what is in fact an upgrade to the existing feature with more robust functions for the purpose. The e-forum 
was developed to aid the operation of CoPs particularly with regard to the sharing of knowledge. These are discussed in detail in subsequent sections.

Company A's existing practice to capture reusable project knowledge from routine weekly-bi-weekly project meetings was consistent with the Capri.net methodology. Moreover, the Capri.net system had provided a Web-based tool where knowledge identified from the routine meetings can be captured in a format complete with direct access to relevant files, photos, condition for reuse, and other background information. This was not possible through Company A's existing practice of recording the knowledge in minutes of meeting. In addition, the robust search function and Webbased access can provide 'live' access and also ease the identification of relevant reusable knowledge captured. What is required is for the project manager or other designated person to enter the knowledge captured into the system. Trials using the original Capri.net system at Company A's weekly site meetings also found that the project team members did not see this workload as significant or unacceptable. This addressed the concern of some staff that significant additional workload is not desired and confirmed the feasibility of the new system.

[Insert Figure 2 here]

\section{Staff Profile}

Staff profile is also known as the company's skills' yellow pages, people's profile, expert directory, staff directory, etc. It is a Web-based database that contains mainly the contact details of staff. The main function is to facilitate the connection of people with the right knowledge to the people who need the knowledge, particularly the more tacit knowledge which is notoriously difficult to codify. In Company $A$, it is to complement and improve the management of knowledge of who knows what which was found over dependent on individual staff's personal network. 
Company A's staff profile was envisaged to include everyone in the company (i.e. from the top management to the receptionist) as there is no significant cost difference between adding all and a proportion of the staff into the system. It would be the first IT-based KM system to be developed as its development and implementation are rather straight forward. Furthermore, there is no substantial training requirement and the benefits brought about will be visible once the number of completed profiles has reached the critical mass.

Company A's staff profile would contain the following details:

- $\quad$ Name of staff;

- Photo(s);

- Job title(s);

- Department/Section;

- $\quad$ Background (i.e. education and previous work experience);

- $\quad$ Contact details (e.g. email, mobile number, phone number/extension and fax);

- $\quad$ Projects involved in (Project title and link to the project website);

- $\quad$ Expertise (e.g. design of pharmaceutical facility);

- $\quad$ Membership of CoPs;

- $\quad$ Level of experience in certain areas;

- Miscellaneous (e.g. languages spoken and interest); and

- $\quad$ Links to knowledge captured in the Capri.net system. 
A robust search function based on a combination of different criteria is to be provided. To ensure that the information in the system is up-to-date, the staff would be required to update their profile at intervals determined by the human resource department.

\section{Custom-designed e-Forum for CoPs}

An e-Forum provides a virtual space for the members of a CoP to exchange their ideas and to interact with each other without being constrained by location and time. In this regard, the experience of Buckman Lab which had quite successfully used Web-based forum applications to link its various CoPs in order to facilitate global knowledge sharing across its regional offices (Pan and Leidner 2003) can be adapted. Buckman Lab's forum had 20 sections (e.g. Pulp and Paper, and Leather), each with its own message board, conference rooms to facilitate debate, and a library section where the communication threads and other pertinent knowledge were stored. Knowledge sharing for Company A's CoPs had been confined to either within a practice in the same department or within the senior staff from a practice across different departments (i.e. through the CoP's meeting). The creation of an e-forum, as evident by the Buckman Lab's experience, will help to enhance the communication of a CoP across all ranks and departments and even allow cross CoPs knowledge creation to happen.

Each section that belongs to different CoPs in Company A would have its own moderator(s) to oversee the activities of the e-forum. The moderator was responsible for the following:

- $\quad$ Ensuring that the messages/questions posted are responded to appropriately and timely. A standard response time of about 2 working days is recommended. This is because if people do not receive any response within a reasonable period of time, it defeats the purpose of creating the system; and 
- Helping to re-direct the questions posted, or the person requesting for assistance, to the 'right' person within the CoP or even in other CoPs as necessary. This task might be assisted by the staff profile system that targets the knowledge about who knows what in the company.

The moderator was given the authority to assign the task to compile an article for the knowledge created through the discourse in the e-forum to an internal expert, who will then upload it into the Capri.net system in order to disseminate to wider audience in a timely manner.

\section{Implementation Strategy}

Depending on the speed at which the benefits of introducing a KM system or practice can be accrued and also the time-frame for implementation, the goals were strategically distinguished as either short-term or long-term. Long-term goal was concerned with the creation of a culture that is supportive of knowledge sharing, which is known to be a very time consuming task. Whereas, shortterm goals include the development and introduction of new IT tools and the integration of Capri.net system with the Company A's other systems according to the sequence of development. Development of an IT tool, for instance Staff Profile, can be accomplished within one month with another month needed for training purpose. This will be followed by data entry which can be completed within a few days. After that, the staff can already start enjoying the convenience and benefit of locating the right people for the right knowledge offered by the Staff Profile system. Therefore, the software tools would be developed one at a time in view of the existing workload of the IS department, whereas the cultivation of a supportive knowledge sharing culture is an on-going and continuing task.

It was envisaged that one of the greatest challenges was to get the buy-in of all the staff which was interwoven with the nurture of knowledge sharing culture, as some had previously not benefited from the company's KM practice. For this, a strategy was formulated for rolling out new IT tool and 
KM practice within Company A. This covered making the announcement in various forms, continuously broadcasting about it, explaining to the members of staff the rationale for having the new tool/practice and how they can benefit from it, as well as providing necessary training and support for using it. Company A had incorporated KM into the company's major events wherever possible. For instance, in the annual leadership day which is an internal seminar culminating what Company A had achieved in the past one year, successful knowledge sharing stories that led to positive outcomes were shared and celebrated in the event. In a way, it is very much like an intensive marketing of KM. As Staff Profile would be the first IT tool to be rolled out, it was part of the plan that CEO would be the first few to have his complete profile in the system to re-emphasize the top management's commitment on the implementation of KM to the staff.

Human resource department was requested to ensure that new recruits are made aware of the company's policy towards knowledge sharing. While being aware that recognition from peers as an expert for being able to share knowledge to help others solving their problem is a motivator for continuing to do so, Company A also planned to give some tangible but non-monetary rewards (e.g. a mug with company's logo) to the staff to strengthen the impact and to encourage more staff to participate. The ultimate goal was to make knowledge sharing part of the Company A's culture.

\section{Potential Impacts and Expected Benefits of the New System}

This study aims to integrate Company A's existing/new KM systems and practice with Capri.net methodology to improve its management of important reusable project knowledge. The fully integrated system comprises mainly a Web-based staff profile, custom-designed e-forum and the Capri.net system, when fully implemented, is expected to have the following impacts and benefits:

- Compared to its existing practice where knowledge is captured but not always easy or possible to locate, the integrated system allows a construction company's knowledge to be fully 
exploited by being able to identify what it knows, where the knowledge is and who knows what effectively and quickly. In addition, the new system ensures that the knowledge is captured together with the critical details to facilitate reuse;

- Knowledge loss is minimized whilst completeness of important reusable project knowledge captured from projects is improved. The capture of project knowledge from routine project meetings and the Capri.net system work in tandem throughout the lifecycle of a project ensuring that no critical knowledge will be lost due to the lapse of time for its capture. This provides also invaluable materials for training and learning purposes;

- The new system integrates the previously isolated and standalone KM systems and practice, leading to a synergized system capable of offering more than the sum of what the individual systems could afford. The access to one of the components of the integrated system will also open the user to other relevant sections. For instance, Staff A encounters a technical problem and posts a request for assistance in the e-forum. Staff B, who is based at another branch, responds to Staff A's request for assistance and provides Staff $A$ the hyperlink to an article in the Web-based knowledge base which is written by Staff $C$ on how to solve a very similar problem a year ago. Staff $A$, after reading through the article in the knowledge base, would like to find out more details of the approach adopted by Staff C. There is a hyperlink in the article to the author's (i.e. Staff C's) "staff profile". Through the hyperlink, Staff A finds out the contact details of Staff C. Staff A proposes to have a video-conference with Staff $C$ for the purpose. Staff $C$ agrees and subsequently helps Staff $A$ to solve the technical problem. Staff A compiles the learning and uploads it into the Web-based knowledge base for company-wide sharing. If other staff in the company encounters similar problem in the future, the potential solution is likely to be located through a search in the e-forum and also the knowledge base. Again, if further assistance is needed the contact details of the experts can be found in the staff profile; 
- It improves the access to knowledge through the integrated system, particularly for the lower-ranked staff who previously were not in the position to attend the CoPs' meeting and were not involved in Company A's other KM activities. Furthermore, the advent of Staff Profile will provide a means to access who knows what in a degree unrivalled by any staff regardless of the length of their service in the company. As the Staff Profile is meant for connecting the right people with the right knowledge, it marks an improvement in managing the more tacit knowledge that counts on people-to-people interaction for its sharing in Company A;

- $\quad$ The purpose served by the integrated system is inline with Company A's strategy to become market leader in its focused sectors for being able to offer value-added solution, which entails a very efficient way of managing its knowledge. Value added solution may cover innovations and creative ideas from internal experts (if one knows who to speak to), or created through the interactions and discussions, which can be facilitated by the new system. It will help to transform the company from a typical construction company to a value-added solution provider; and

- The new system will be an integral component of Company A's plan to nurture a knowledge sharing culture. Knowledge sharing culture and the integrated system is interdependent. This is simply because in order to have at least a continuous satisfactory level of knowledge sharing activities in any KM system, the existence of a supportive knowledge sharing culture will prove to be essential. However, for a knowledge sharing culture to be nurtured in Company A with about 800 staff, a KM system must be in place to make it easy for knowledge sharing so that it will not be treated as an unpleasant task.

Even though the new integrated system was developed for Company A and based on the research conducted with the company, the concepts and principles developed can readily be adopted or adapted by other similar construction sector companies. The core of the framework - the Capri.net system, was well received in an evaluation by staff from the collaborating construction companies due to its ease of use and its ability to capture the details of reusable project knowledge. The other 
components of the framework, namely the e-forum and staff profile system, are commonly used tools to facilitate better knowledge sharing among their staff across geographical boundaries with proven track records. This framework has successfully integrated Capri.net and two other commonly-used systems and added some essential new features. The successful adoption of the framework is considered feasible albeit necessary adaptation will be needed to customize it to suit the different context of different companies.

\section{Potential Limitations and Shortcomings of the New System}

As the new system is an integration of a few standalone information systems, this makes the powerful search function a very important component. If compared with Google's search engine, there is no function available in the new system that can automatically suggest words or phrases to enhance the result of the search. The existing search function also does not provide a summary of the results returned, which if available, might be helpful in giving the users a better view of the relevance of the items in the result list. In addition, revisions might be made to the knowledge captured in the system as necessary. However, the current system does not have an audit trail function to keep track of the revisions made to a knowledge item. These are the potential limitations that might be addressed in the development of an updated version of the system.

\section{Conclusions}

This paper investigated the integration of the methodology for 'live' capture and reuse of project knowledge (i.e. Capri.net) with Company A's other KM systems and practice to enhance the management of knowledge created from its ongoing projects. The study revealed that the Capri.net methodology has the potential of serving as the central KM system for capturing explicit project knowledge and facilitating the sharing of tacit knowledge with the aid of staff profile and custom- 
designed e-forums for CoPs. The staff profile provided an easily accessible channel to the knowledge of who knows what covering potentially everyone in a company to bridge the communication gap for knowledge sharing. The e-forum enabled the exchange of ideas and the interactions among staff without the constraints of location of time. The significance of a staff profile and e-forum in knowledge management had been widely acknowledged in the existing literature. However, the integration of the staff profile and e-forum with the Capri.net methodology could lead to a synergy which is larger than the sum of what the individual components could achieve individually. For instance, the link to the staff profile of an expert who tries to help another staff in solving a technical problem through e-forum will enable the latter to find out other more effective means of communicating with the expert on the matter.

The general perception that the implementation of $\mathrm{KM}$ in construction companies entails a large amount of monetary investment or an extensive reengineering of existing business processes and practices may need revision. As evident in this study, it might only involve the development or upgrading of some existing IT systems to integrate with an organization's practice (with necessary adaptation) for capturing important project knowledge. The most challenging task was to obtain the buy-in of the staff and to nurture the desired knowledge sharing culture. Phasing the implementation of KM can be done if there is constraint in certain resources, and to produce real evidence to justify the need for more resources and to secure the buy-in of the staff.

This research has demonstrated that a top-down approach to the implementation of KM in Company $A$ is a viable option. This is different from the typical bottom-up initiative where $\mathrm{KM}$ is proposed by middle managers, who have to get the business case prepared and approved, and obtain the necessary resources for the purpose. The top-down approach, if properly executed and coupled with a pragmatic strategy, was found to help curb potential resistance from staff. The shaping of a supportive knowledge sharing culture and the ability to gain the cooperation from the staff require 
the commitment from the top to be in place and clearly visible. With this, staff buy-in to the KM system may follow subsequently when they have personally benefited from it. 


\section{References}

Al-Ghassani, A. M. (2002). Literature Review on KM Tools, Department of Civil and Building Engineering, Loughborough University, UK.

Carrillo, P. M., Robinson, H. S., Al-Ghassani, A. M. and Anumba, C. J. (2002). Survey of Knowledge Management in Construction, Department of Civil and Building Engineering, Loughborough University, UK.

Chinowsky, P. and Carrillo, P. (2007). "Knowledge Management to Learning Organization Connection." J. Manage. in Eng., 23(3), 122-130.

CIPD (2010). Annual survey report 2010: Resourcing and talent planning, Chartered Institute of Personnel and Development.

Dalkir, K. (2007). Knowledge management in theory and practice, Butterworth-Heinemann, Oxford.

Ebbinghaus, H. (1885). Uber das Gedachtnis, Leipzig, Dunker. Translated by Rugger, H. A. and Bussenius, C. E. (1913), Teachers College, Columbia University, Canada. <http://psychclassics.yorku.ca/Ebbinghaus/index.htm> (Aug. 16, 2004).

Fang, Y. and Chiu, C. (2010). "In justice we trust: Exploring knowledge-sharing continuance intentions in virtual communities of practice." Computers in Human Behavior, 26, 235-246.

Health and Safety Executive (HSE) (2007). "Managing health and safety in construction: Construction (Design and Management) Regulations 2007 Approved Code of Practice." Health and Safety Executive, UK.

Kamara, J. M., Anumba, C. J., Carrillo, P. M. and Bouchlaghem, N. M. (2003). “Conceptual Framework for Live Capture of Project Knowledge." Proc., CIBW078 International Conference on Information Technology for Construction, CIBW078, Waiheke Island, New Zealand, 178- 185. 
Kartam, N. A. (1996). "Making effective use of construction lessons learned in project life cycle." J. Constr. Engr. and Manage., March, 14-21.

Kebede, G. (2010). "Knowledge management: An information science perspective." International Journal of Information Management, 30, 416-424.

Kerth, N. (2000). "The ritual of retrospectives: How to maximise group learning by understanding past projects." Software Testing \& Quality Engineering, September/October, 53-57.

Kotnour, T. and Vergopia, C. (2007). “A Framework and Findings for Learning Based Project Reviews." Proc., PICMET 2007, Portland, Oregon.

Nguyen, Q. T. N., Neck, P. A. and Nguyen, T. H. (2009). "The Critical Role of Knowledge Management in Achieving and Sustaining Organisational Competitive Advantage." International Business Research, 2(3), 3-16.

Linton, M. (1975). "Memory for real-world events." Explorations in Cognition, D. A. Norman and D. E. Rumelhart, eds., W. H. Freeman, San Francisco.

McGee, K. G. (2004). Heads Up: How to anticipate business surprises and seize opportunities first, Harvard Business School Press, MA.

Newell, S., Robertson, M., Scarbrough, H. and Swan, J. (2002). Managing Knowledge Work, Palgrave, New York.

Nonaka, I. and Konno, N. (1998). "The Concept of "Ba": Building Foundation for Knowledge Creation." California Management Review, 40(3), 40-54.

Nonaka, I. and Takeuchi, H. (1995). The Knowledge-Creating Company: How Japanese Companies Create the Dynamic of Innovation, Oxford University Press, Oxford. 
O'Dell, C. and Grayson, C. J. (1998). "If only we knew what we know: Identification and transfer of internal best practices." California Management Review, 40(3), 154-174.

Orange, G., Burke, A. and Cushman, M. (1999). “An approach to support reflection and organisation learning within the UK construction industry." Proc., BITWorld'99, Capetown, SA.

Pan, S. L. and Leidner, D. E. (2003). "Bridging Communities of Practice with Information Technology in Pursuit of Global Knowledge Sharing." J. Strategic. Info. Sys., 12, 71-88.

Plessis, M. (2007). "The role of knowledge management in innovation." J. of Knowl. Manage., 11(4), 20-29.

Robinson, H.S., Carrillo, P.M., Anumba, C.J. and Bouchlaghem, N.M. (2004). Investigating Current Practices, Participation and Opportunities in the Private Finance Initiatives (PFI): A Survey of Construction and Client Organisations, Department of Civil and Building Engineering, Loughborough University, UK.

Saint-Onge, H. and Wallace, D. (2003). Leveraging Communities of Practice for Strategic Advantage, Butterworth-Heinemann, Oxford.

Schindler, M. and Eppler, M. J. (2003). "Harvesting project knowledge: A review of project learning methods and success factors." Int. J. Project Manage., 21, 219-228.

Trussler, S. (1999). "The Rules of the Game." The Knowledge Management Yearbook 1999-2000, J.W. Cortada and J.A. Woods, eds., Butterworth-Heinemann, Boston, 280-286.

Wenger, E. (2004). Communities of practice: learning, meanings, and identity, Cambridge University Press, Cambridge.

Whetherill, M., Rezgui, Y., Lima, C. and Zarli, A. (2002). "Knowledge management for the construction industry: The e-COGNOS Project." ITcon, 7, Special Issue: ICT for Knowledge Management in Construction, 183-196. 
Table 1: Background of Case Study Companies

\begin{tabular}{|c|c|c|c|c|}
\hline Company & Company background & $\begin{array}{l}\text { Background of the } \\
\text { interviewees }\end{array}$ & $\begin{array}{l}\text { Number of } \\
\text { employees }\end{array}$ & $\begin{array}{l}\text { Annual revenue } \\
\text { (f) }\end{array}$ \\
\hline$A$ & $\begin{array}{l}\text { Construction, Property } \\
\text { Development, and Design } \\
\text { Consultant }\end{array}$ & $\begin{array}{l}\text { Managing Director, IT } \\
\text { Manager, Systems } \\
\text { Manager \& Procurement } \\
\text { Manager }\end{array}$ & 850 & f250M \\
\hline B & Design Consultant & $\begin{array}{l}\text { Partner, Associates \& IT } \\
\text { Manager }\end{array}$ & 80 & f4.3M \\
\hline C & Engineering Consultant & $\begin{array}{l}\text { Group Knowledge } \\
\text { Manager \& Knowledge } \\
\text { Researcher }\end{array}$ & 7000 & $£ 403 \mathrm{M}$ \\
\hline D & Management Consultant & $\begin{array}{l}\text { Group Knowledge } \\
\text { Manager, Associate } \\
\text { Director \& Head of R\&D }\end{array}$ & 1200 & $f 61 M$ \\
\hline $\mathrm{E}$ & $\begin{array}{l}\text { Project Extranet Service } \\
\text { Provider }\end{array}$ & $\begin{array}{l}\text { Director of Business } \\
\text { Development, Senior } \\
\text { Account Manager \& } \\
\text { Senior Customer Support } \\
\text { Staff }\end{array}$ & 31 & $f 2 \mathrm{M}$ \\
\hline $\mathrm{F}$ & Water Company & Knowledge manager & 18000 & f1860M \\
\hline
\end{tabular}

